\title{
The Case Study of Pseudoexcitation Method Combining Self-Adaptive Gauss Integration in Random Vibration Analysis
}

\author{
Ning Chen, ${ }^{1}$ Siyu Zhu ${ }^{(D)}{ }^{2}$ and Yongle Li ${ }^{3}{ }^{3}$ \\ ${ }^{1}$ School of Civil Engineering, Hunan University of Science and Technology, Xiangtan, Hunan 411201, China \\ ${ }^{2}$ College of Environment and Civil Engineering, Chengdu University of Technology, 610059 Chengdu, Sichuan, China \\ ${ }^{3}$ Department of Bridge Engineering, Southwest Jiaotong University, 610031 Chengdu, Sichuan, China
}

Correspondence should be addressed to Siyu Zhu; blueskyzsy@aliyun.com

Received 19 August 2019; Revised 3 November 2019; Accepted 11 November 2019; Published 26 November 2019

Academic Editor: Vadim V. Silberschmidt

Copyright (C) 2019 Ning Chen et al. This is an open access article distributed under the Creative Commons Attribution License, which permits unrestricted use, distribution, and reproduction in any medium, provided the original work is properly cited.

The pseudoexcitation method (PEM) can improve efficiency of random vibration analysis. However, for large-sized structures with wide frequency range of response, the workload of calculation is heavy if conventional integration methods, such as trapezoidal integration, are used to combine with the PEM to calculate structural response. In such case, self-adaptive technology is induced to combine with the PEM to form an efficient method for solving random vibration. During calculation, this method can realize the adaptability of random excitation to actual structural response, identify automatically critical frequency intervals of random excitation, and process intelligently the identified critical frequency intervals and noncritical frequency intervals. Based on the identified frequency intervals, Gauss integration is carried out to obtain response results with random characteristics. The computational efficiency and accuracy of PEM-SGI are verified by wind-induced performance of the slender bridge tower. Finally, the influence of damping ratio of the bridge structure and train marshalling on vehicle-bridge coupled system is investigated to further verify the application of the proposed method. Results show that the efficiency of solving random vibration can be improved by the present method.

\section{Introduction}

Random vibration, as one of the most important topics in the field of structure engineering, has experienced abundant research progress [1]. The huge computational workload, however, often becomes bottleneck which limits its application in practical engineering. In such case, many scholars have been keeping efforts and obtained fruitful achievements. Zhu reviewed the recent developments and applications of the stochastic averaging method in random vibration [2]. Kiureghian proposed a procedure to investigate a certain structure subjected to seismic excitations [3]. Türkay et al. investigated the response of the vehicle to profile-imposed excitation with randomly varying traverse velocity and variable vehicle forward velocity based on the quarter-car model [4]. Zhang et al., based on the theory of the pseudoexcitation method, proposed an optimized methodology to investigate the seismic analysis of multisupported structure subjected to spatially varying ground motions [5]. Soyluk applied spectral analysis approach and two response methods to investigate the spatial variability effects of ground motion of the dynamic behavior of long-span bridges [6]. Kareem presented some results of addressing issues concerning the dynamic response of highrise buildings with stochastic wind loads [7]. Benfratello et al. investigated the role of the quadratic term of the forcing function in the response statistics of the multidegree-offreedom wind-excited linear-elastic structure [8]. $\mathrm{Li}$ and Kareem studied the dynamic behavior of the tension-leg platform under the simultaneous action of random wind and wave fields [9]. Le and Caracoglia applied the waveletGalerkin method to investigate the nonlinear stochastic dynamic system, and a slender building is selected as an example to study the coupled response by transient wind load [10].

Among them, the pseudoexcitation method proposed by Professor Lin, greatly improves computational efficiency while guaranteeing high accuracy [11-13]. At present, the 
pseudoexcitation method has been widely used in many engineering fields [14-17] and has gradually become one of the most important numerical methods for random vibration analysis and dynamic reliability analysis. Besides, the random vibration of the vehicle-bridge has obtained much more attention. Coussy et al. [18] applied means of a theoretical study to analyze the effects of random surface irregularities upon the dynamic response of bridges subjected to moving loads. Rocha et al. [19] used a probabilistic methodology to investigate the safety assessment of a shortspan railway bridge for high-speed traffic. Zeng et al. [20] presented the formulation of a three-dimensional equation of motion for a train-track-bridge coupling system, and its application to random vibration analysis is also expressed. Yu et al. [21] proposed a new random vibration theory to study the stochastic response of the coupled train-bridge systems. Yin et al. proposed a new approach to study the nonstationary random response of the bridge under moving vehicles with variable speed [22]. Mao et al. presented a new random method for railway dynamic simulation to investigate the dynamic responses of a $3 \mathrm{D}$ train-bridge coupled system involving random parameters of the system [23]. Wang et al. analyzed the dynamic behavior of the bridge under traffic loadings and vehicle dynamic interactions $[24,25]$.

For random vibration analysis, as a large number of discrete frequency points should be extracted to calculate the response power spectrum, numerical integration techniques, such as trapezoidal integration, are used to obtain the variance of structural response. However, for large-sized and complex structures with a wide frequency response range, if trapezoidal integration is applied to calculate the variance of structural response, the number of discrete frequency points would be excessive, which greatly increases the computational workload. Compared with trapezoidal numerical integration, Gauss numerical integration that has many advantages, such as fewer integral numbers needed and higher integration accuracy, has been widely used in various engineering numerical calculations [26-28].

In this paper, self-adaptive Gauss integration (SGI) is introduced into the pseudoexcitation method (PEM) to form a high-efficient combination method (PEM-SGI). Its basic idea is to realize the self-adaptability of response results to random excitation through adaptive iteration. During calculation, the calculation program can identify automatically the location of frequency points that demonstrate critical effects of random excitation on the response of the structural system [29]. Meanwhile, intelligent processing is carried out for the identified critical and noncritical frequency intervals to find reasonable integral subintervals, and all the subintervals are solved by Gauss integration. Since the hybrid method is based on the theory of pseudoexcitation method, it is just suitable to handle dynamic response analysis of the linear or weak nonlinear structure with external random excitation. In this paper, some case studies, which are focused on the stationary random loads, are applied to express the applicability and efficiency of the PEM-SGI. Firstly, the numerical model of a super-high bridge is selected, and the calculation is carried out with the influence of Davenport's power spectrum density function of fluctuating wind load. Based on the wind-induced vibration of the bridge tower, the PEM-SGI can be verified and the computational efficiency is explained. Then, the singlemarshalling high-speed train model with 23 degree of freedom is taken as an example to simulate the whole process of the train crossing the bridge. The influence of the damping ratio of the bridge and train marshalling on the vehiclebridge coupled system is investigated.

\section{Pseudoexcitation Method for Stationary Random Excitation [11, 12, 15]}

The main feature of the pseudoexcitation method is to transform stationary random response analysis into a series of steady-state harmonic response analysis. Taking stationary random vibration with multiple degrees of freedom as an example, this paper elaborates the basic ideas of this method. The structural dynamic equation with multiple degrees of freedom can be written as

$$
[M]\{\ddot{y}\}+[C]\{\dot{y}\}+[K]\{y\}=[E]\{f(t)\},
$$

where $[M],[C]$, and $[K]$ are the mass, damping, and stiffness matrix of the structure, $\{f(t)\}$ is the excitation vectors, $[E]$ is the indicator matrix of excitation vectors, and $\{y\},\{\dot{y}\}$, and $\{\ddot{y}\}$ represent the displacement, velocity, and acceleration of structural response, respectively.

When the external excitation $\{f(t)\}$ is a stationary random process with normal distribution, assuming the autopower spectral density function as $\left[S_{f f}(\omega)\right]$, the frequency response function matrix $[H(\omega)]$ is given; meanwhile, the autopower spectral density function of response can be written as

$$
\left[S_{y y}(\omega)\right]=[H(\omega)]^{*}\left[S_{f f}(\omega)\right][H(\omega)]^{T},
$$

where superscript $*$ and $T$ denote the complex conjugate and transpose of the matrix to be solved.

The autopower spectral density matrix of excitation $\left[S_{f f}(\omega)\right]$ is decomposed by Cholesky as

$$
\left[S_{f f}(\omega)\right]=[L][L]^{T}=\sum_{k=1}^{m}\{l\}_{k}\{l\}_{k}^{T}
$$

where $m$ represents the number of decomposed matrix, $[L]$ is the triangular matrix, and $\left\{l_{k}\right.$ is the Kth column vector of the matrix $[L]$. Substituting formula (3) into formula (2) gives

$$
\left[S_{y y}(\omega)\right]=\sum_{k=1}^{m}[H(\omega)]^{*} \cdot\{l\}_{k} \cdot\{l\}_{k}^{T} \cdot[H(\omega)]^{T} .
$$

Construct $m$ independent pseudoharmonic excitations as

$$
\begin{aligned}
\{\tilde{f}\}_{k} & =\{l\}_{k} e^{i \omega t}, \\
k & =1 \sim m,
\end{aligned}
$$

for each pseudo excitation $\{\tilde{f}\}_{k}$, the deterministic steady response of the structure is 


$$
\{\tilde{y}\}_{k}=[H(\omega)]\{l\}_{k} e^{i \omega t} .
$$

Compared with formula (4), the power spectral density function of response can be expressed as

$$
\left[S_{y y}(\omega)\right]=\sum_{k=1}^{m}\{\tilde{y}\}_{k}^{*} \cdot\{\tilde{y}\}_{k}^{T} .
$$

Calculation procedures mentioned above are the basic ideas of the pseudoexcitation method.

The variance of response can be obtained by integrating the power spectral density function of response:

$$
\sigma_{y, i}^{2}=\int_{-\infty}^{+\infty} S_{y y, i}(\omega) \mathrm{d} \omega
$$

where $S_{y y, i}(\omega)$ is the $i$ th principal component of the power spectral density matrix of response $\left[S_{y y}(\omega)\right]$ and $\sigma_{y, i}^{2}$ is the response variance corresponding displacement. Trapezoidal integration can be used for numerical solution of formula (8).

\section{Introduction to PEM-SGI Methodology}

In order to obtain the variance of structural responses in random vibration analysis, it is often necessary to calculate the time-varying power spectrum of response for a large number of discrete frequency points and to integrate the power spectrum with numerical integration technology to obtain response variance. Although the pseudoexcitation method can improve significantly the computational efficiency of random vibration analysis, for large-sized complex structures with a wide frequency response range, the computational workload will be large as a result of excessive discrete frequency points, if the structural response variance is calculated by the conventional integration method. Compared with trapezoidal numerical integration, Gauss numerical integration owing advantages of fewer integral numbers needed and higher integration accuracy is widely applied into numerical calculation for various engineering fields $[30,31]$. The self-adaptive Gauss integration is introduced into the pseudoexcitation method, the basic idea of which is to find reasonable integral subintervals of frequency intervals by self-adaptive iteration and to solve each subinterval by Gauss integration.

When the vehicle-bridge coupling system is calculated, integral functions are more complex and most of them are implicit. Even if explicit integral functions can be written, the integration is extremely complex and numerical integration is thus often used to replace the definite integration of functions. Numerical integration means that some points called integral points are selected according to certain rules in the integral region. Based on these selected integral points, the corresponding results of integral functions are solved and then multiplied by corresponding weighted coefficients and summed up as approximate integral values. The commonly used numerical integration methods are the rectangular method, trapezoidal method, and Simpson method, which are all limited to set equal points in the selected intervals as integral points and to approximate calculate the integrand $\quad y(x)=a_{m} x^{m}+a_{m-1} x^{m-1}+a_{m-2} x^{m-2}+\cdots+a_{0}$ by $m$ order polynomials. The rectangular method is constant approximation; trapezoid formula, with accuracy of the firstorder algebra, is linear approximation; and Simpson formula, with accuracy of the third-order algebra, is parabolic approximation. For Gauss integration, without limits to use equal points as integral numbers, higher accuracy or the same accuracy can be achieved with the same integral numbers or fewer integral numbers. Numerical integration methods that are commonly used are the analytical and computational method in engineering science, among which Gauss numerical integration has the advantages of less integral numbers and higher computational accuracy. The basic formula is

$$
\int_{a}^{b} f(x) \mathrm{d} x=\sum_{j=1}^{n} A_{j} f\left(x_{j}\right),
$$

where $A_{j}(j=1 \sim n)$ is the quadrature coefficient independent of $f(x)$ and $x_{j}(j=1 \sim n)$ is the integral point. Proper selection of $A_{j}$ and $x_{j}$ can make formula (9) have accuracy of $2 n-1$ order algebra.

The value of the weight factor $A_{j}$ and the integral point $x j$ of the Gauss integration formula in the integral interval $[-1,1]$ are given in the previous literature [27]. When integral numbers are 2 , the integral point coordinates are $x_{1,2}= \pm 0.577350$ and the weight factor is $A_{1,2}=1,1$. For integration in any interval shown in formula (9), it can be solved by transforming it to the interval of $[-1,1]$. It is obvious that it is difficult to obtain satisfactory results by integrating the whole interval with Gauss numerical integration in formula (8). It is more reasonable to divide the integral interval into several subintervals and to integrate each subinterval separately with the Gauss numerical method. Therefore, how to divide Gauss integration subintervals is very important for improving the accuracy and efficiency of computation. In such case, self-adaptive Gauss integration technique is induced to solve this problem [29].

Therefore, this program can determine automatically the convergence of results and decompose functionally frequency intervals to get final results. It realizes the automatic identification of frequency intervals which demonstrate significant and insignificant influence of random excitation on the stochastic characteristics of the system in the selfadaptive optimization method and also realizes the functionality to subdivide intelligently important frequency intervals. The frequency interval in which final convergence results can be obtained only after several subdivisions is the important interval of random excitation for random vibration of the vehicle-bridge coupled system. What does not need to be subdivided is insignificant frequency intervals, but be roughly segmented or discarded directly, thus reducing computational cost and improving computational efficiency. Meanwhile, researchers can choose $\varepsilon$ flexibly according to the requirements of engineering and research projects and adjust the target error to find the balance point of computational efficiency and accuracy that meets actual needs. 


\section{Verification and Application of PEM-SGI Technique}

4.1. Random Wind-Induced Vibration Analysis of Slender Bridge Tower. Taking the super-high bridge tower of a cablestayed bridge as an example, structural wind-induced random response subjected to fluctuating wind is analyzed. The structure of the bridge tower is shown in Figure 1. The total height of the bridge tower is $398.16 \mathrm{~m}$. The pylon column is a linearly variable cross section along the height direction. The bridge tower adopts C50 concrete, the elastic modulus is $3.45 \times 10^{4} \mathrm{MPa}$, Poisson's ratio is 0.2 , the damping ratio is 0.02 , and the density is $2549 \mathrm{~kg} / \mathrm{m}^{3}$.

For calculation, the wind load power spectrum proposed by Davenport is used [32]:

$$
S_{F}(\omega)=16 \cdot k \cdot \bar{F}^{2}(z) \cdot\left[\frac{\bar{v}_{10}}{\bar{v}(z)}\right]^{2} \times \frac{1}{\omega} \cdot \frac{\left(600 \omega / \pi \cdot \bar{v}_{10}\right)^{2}}{\left[1+\left(600 \omega / \pi \cdot \bar{v}_{10}\right)^{2}\right]^{3 / 4}}
$$

where $\bar{v}_{10}$ the mean wind speed at the height of $10 \mathrm{~m}$, in the present paper, is $40 \mathrm{~m} / \mathrm{s}, \bar{v}(z)$ is the mean wind speed at the height of $z$, and $k$ is the surface roughness coefficient, which is 0.03 in this paper. $\omega$ is the circular frequency of excitation. When analyzing the pseudoexcitation, a single-side power spectrum adopted as the upper limit of $\omega$ integration is $30 \mathrm{rad} / \mathrm{s}$ and the lower limit is $0 \mathrm{rad} / \mathrm{s} . \bar{F}(z)$ is the mean wind load acting on the height of $Z$, and the expression is as follows:

$$
\bar{F}(z)=\frac{1}{2} \rho \bar{v}(z)^{2} \cdot A(z) \cdot C_{d},
$$

where $\rho$ is the air density, set as $1.226 \mathrm{~kg} / \mathrm{m} 3, A(z)$ is the windward area at the height of $Z$, and $C_{d}$ is the drag coefficient.

The mean wind speed changing along the height adopts the exponential equation. Formula of $\bar{v}(z)$ and $\bar{v}_{10}$ are given as

$$
\frac{\bar{v}(z)}{\bar{v}_{10}}=\left(\frac{z}{10}\right)^{\alpha}
$$

where $\alpha$ is the roughness index of the bottom, set as 0.12 in this paper.

The cross power spectral density of wind loads at two high points $z_{1}$ and $z_{2}$ is defined as

$$
S_{F F}\left(\omega, z_{1}, z_{2}\right)=\sqrt{S_{F}\left(\omega, z_{1}\right) \cdot S_{F}\left(\omega, z_{2}\right)} \times \rho\left(\omega, z_{1}, z_{2}\right),
$$

where $\rho\left(\omega, z_{1}, z_{2}\right)$ is the coherence function, and it can be written as

$$
\rho\left(\omega, z_{1}, z_{2}\right)=\exp \left(-\frac{C_{1} \cdot \omega}{2 \pi} \cdot \frac{\left|z_{1}-z_{2}\right|}{\bar{v}_{10}}\right),
$$

where the value of $C_{1}$ is 7.0 .

Trapezoidal integration and self-adaptive Gauss integration techniques are used to calculate the variance of lateral displacement response at the top of the bridge tower for random vibration of structures. The results are shown in Table 1. From the table, if the computational accuracy with two valid digits after the decimal point is to be achieved, the calculation interval should be divided into at least 1500 integral subintervals for trapezoidal integration, i.e., the power spectral density $S_{y y}(\omega)$ should be calculated for 1500 discrete frequency points. By self-adaptive Gauss integration, the computational accuracy with two valid digits after the decimal point can be achieved by dividing the calculation interval into 55 subintervals, which is $3.7 \%$ of trapezoidal integration intervals. Only 110 frequency discrete points need to be calculated in the whole self-adaptive process, which is $7.3 \%$ of the computational times in trapezoidal integration. The standard deviation of the top displacement obtained by these two methods converges to $0.5334 \mathrm{~m}$ if the computational accuracy with four valid digits after the decimal point is to be achieved. The number of integral subintervals needed for trapezoidal integration is 2400 , i.e., the number of discrete frequency points calculated by power spectrum is 2500 times. It takes almost 700 minutes to finish the whole computation. But the selfadaptive Gauss integration only costs nearly 5 minutes to gain the results which requires 127 intervals, and the corresponding number of discrete frequency points calculated by power spectrum is 254 times, which is only $10.6 \%$ of trapezoidal integration.

The self-adaptive Gauss integration method is introduced into the pseudoexcitation method of random vibration. By self-adaptive iteration, the integral interval is subdivided into several reasonable integral subintervals and each integral subinterval is separately integrated by the Gauss numerical method, which greatly improves the efficiency of integration calculation. The numerical examples show that the self-adaptive Gauss integration technique can significantly reduce computational times and further improve computational efficiency of the pseudoexcitation method with the same requirements for accuracy. For the random vibration analysis of large-sized practical engineering structures, this method is of practical significance in engineering.

\subsection{Analysis of Random Characteristics of Vehicle-Bridge Coupled System}

4.2.1. Background of PEM-SGI for Vehicle-Bridge Coupled System [29]. PEM-SGI is used to study the random vibration response of the vehicle-bridge coupled system when train passes the three-span simply supported bridge. The length of every span bridge is $40 \mathrm{~m}$ and the total length of the bridge is $120 \mathrm{~m}$, cross-sectional area is $A=9.6632 \mathrm{~m}^{2}$, torsional moment of inertia is $I_{x}=32.75 \mathrm{~m}^{4}$, lateral bending moment of inertia is $I_{y}=87.96 \mathrm{~m}^{4}$, vertical bending moment of inertia is $I_{z}=17.14 \mathrm{~m}^{4}$, the damping ratio of bridge is 0.02 , concrete density is $2600 \mathrm{~kg} / \mathrm{m}^{3}$, and secondary dead load of mass per meter is $7730 \mathrm{~kg} / \mathrm{m}$. The power spectrum of stationary random processes with three kinds of irregular orbits is obtained by the power spectrum density German rail irregularity spectra for high-speed railway. The parameters 


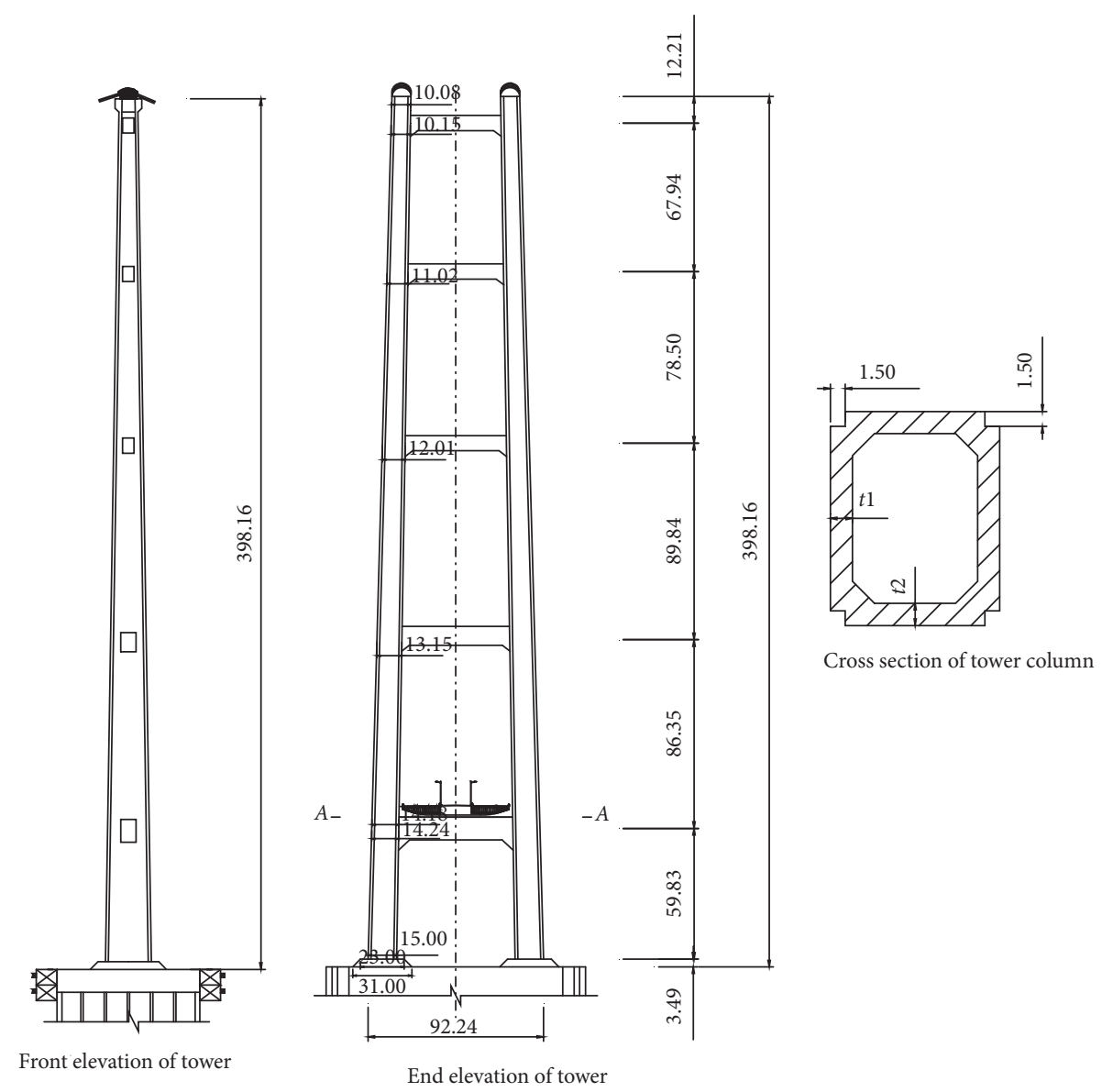

FIGURE 1: Structural arrangement concrete bridge tower (unit: m).

TABLE 1: Random vibration analysis results of the bridge tower.

\begin{tabular}{|c|c|c|c|}
\hline Integration & Subintervals & Standard deviation & Time (minutes) \\
\hline \multirow{8}{*}{ Trapezoidal integration } & 500 & 0.4247 & 145.77 \\
\hline & 1000 & 0.5591 & 291.54 \\
\hline & 1500 & 0.5289 & 437.31 \\
\hline & 1600 & 0.5383 & 466.47 \\
\hline & 2000 & 0.5346 & 583.08 \\
\hline & 2100 & 0.5326 & 612.24 \\
\hline & 2300 & 0.5343 & 670.55 \\
\hline & 2400 & 0.5334 & 699.70 \\
\hline \multirow{4}{*}{ Self-adaptive Gauss integration } & 10 & 0.7690 & 2.92 \\
\hline & 19 & 0.4612 & 5.54 \\
\hline & 55 & 0.5385 & 16.03 \\
\hline & 127 & 0.5334 & 37.03 \\
\hline
\end{tabular}

of the vehicle model are cited in article [29]. For the standard deviation of dynamic response of the vehicle-bridge coupled system, the vertical vibration shape of the vehicle body is selected as the target value for statistical analysis. Therefore, the $X$ axis of all the figures of the bridge response starts with $40 \mathrm{~m}$ when the vehicle steps on the second span of the bridge. The vertical and lateral natural frequencies of the bridge are $4.22 \mathrm{~Hz}$ and $9.53 \mathrm{~Hz}$, respectively. The natural frequencies of the vehicle stay in the range of $0.52 \sim 1.41 \mathrm{~Hz}$, and the natural frequencies of the vehicle body are also presented in Table 2.
In the cited paper [29], the Monte Carlo method is applied to verify the proposed method for the dynamic response of the vehicle-bridge system. For the bridge and vehicle responses, the discrepancy reduced with the increasing of the number of samples.

The Monte Carlo method has been used to validate the PEM-SGI method for random vibration problems of the vehicle-bridge coupled system. Comparing the mean value and standard deviation of PEM-SGI with those of the Monte Carlo method, the results show that the acceleration of the 
TABLE 2: The natural frequencies of the vehicle body (unit: $\mathrm{Hz}$ ) [29].

\begin{tabular}{lcc}
\hline Order & Proposed model & Vibration shape \\
\hline 1 & 0.0000 & Rigid body movement \\
2 & 0.0000 & Rigid body movement \\
3 & 0.7228 & Vehicle body floating \\
4 & 0.8913 & Vehicle body rolling \\
5 & 0.8980 & Vehicle body nodding \\
\hline
\end{tabular}

vehicle body and the bridge structure coincides well, which can meet the requirements for calculation. For the same accuracy of results, the sample number required for PEMSGI is 73 and 246 samples are required for traditional PEM. It reflects the proposed method increases the computational efficiency 3.37 times.

4.2.2. Coupled System with Different Damping Ratio of Bridge. As high-speed of trains will cause the increase of vibration, it is very crucial to reduce vehicle vibration to ensure their smooth running on the bridge. Vehicle vibration includes vibration caused by the track irregularity and the interaction between the vehicle and bridge, among which vehicle vibration caused by the track irregularity is mainly controlled by the power spectrum of track irregularity and dynamic characteristics of the vehicle model. However, vehicle vibration caused by the interaction between the vehicle and bridge structure is determined by inherent characteristics of the vehicle model and bridge structure. It is difficult to correct relevant parameters of the vehicle model and cannot change effectively the inherent characteristics of vehicle model. But the damping ratio of the bridge structure can be modified by adding the damper, so as to change the dynamic vibration of the bridge structure. In this section, numerical calculation and analysis, therefore, are mainly conducted for the influence of structural damping ratio of the bridge structure on the random vibration of the vehicle-bridge coupled system.

The single-marshalling vehicle was selected as the vehicle model, with a running speed of $250 \mathrm{~km} / \mathrm{h}$ and step length of calculation is $0.004 \mathrm{~s}$. Comparing the damping ratio 0.02 and 0.05 , respectively, of the bridge structure, the target value of the bridge is set as response results of the center point in the second span. The random response of the vehicle-bridge coupled system is mainly caused by track irregularity. The vehicle vibration is mainly influenced by track irregularity and inertial force generated by vibration of the bridge structure. In the present calculated condition, the large stiffness of the bridge structure makes natural frequency of bridge vibration far away from that of vehicle model vibration. So generated vibration of the bridge structure is considered as the equivalent track irregularity within the excited vibration of frequency range; meanwhile, the equivalent track irregularity cannot affect the dynamic performance of the vehicle model and the coupled effect between vehicle and bridge therefore cannot be obvious. As a result, different damping ratios of bridge structure will not have a significant impact on response variance of the vehicle body. Structural vibration is mainly divided into forced vibration under external loads and free vibration without load. The different damping ratios of the structure cannot change vehicle dynamic characteristics, but it can effectively control the free vibration of the bridge structure. Therefore, the results of the bridge are expressed in Figure 2, but the vehicle is not exhibited. It can be seen from the figure that the increase of the damping ratio of the structure has a significant impact on response of the bridge structure.

With the increase of damping ratio of the structure, response of the bridge structure significantly decreases. When the vehicle travels in the interval of $40 \sim 60 \mathrm{~m}$, which is the whole process from the train step on the target span of the bridge, there is no obvious change in the response of the bridge structure and results are shown in Figure 2. It shows that when the bridge structure is suddenly subjected to an external load in the process of gradual appearing of vibration, the influence of damping ratio on the vibration of the bridge structure is slight. However, when the vehicle body is on the bridge and even running through the bridge, the damping ratio will have effects since all vertical responses of the bridge structure are greatly reduced and also the gap between these two working conditions is gradually widened. The gap between these two working conditions in the lateral response of the bridge structure gradually widens after the vehicle is on the bridge (60 80 m) and after the vehicle is off the bridge completely (95 140 m). Therefore, the increase of structural damping ratio can reduce the response variance of the bridge structure effectively. However, for the vehiclebridge coupled system model selected in this paper, as vibration of the bridge structure cannot be applied to the vehicle model effectively, the increase of damping ratio of the bridge structure cannot control or reduce the dynamic response of the vehicle model availably. Therefore, measures to control dynamic vibration of the vehicle-bridge coupled system can be selected after analyzing actual response of the specific structure, thus meeting the practical requirements of engineering projects.

\subsubsection{Coupled System with Different Vehicle Marshalling.} In order to reflect and be combined with the actual engineering projects, simulation of the whole process of single-, double-, four-, and eight-marshaling vehicle crossing the bridge was conducted to compare the difference between random characteristics of the multiple units (MU) train and that of the single train. The multimarshalling MU trains are $20 \mathrm{~m}$ away from the end of the bridge with a running speed of $250 \mathrm{~km} / \mathrm{h}$ and step is $0.004 \mathrm{~s}$. The first vehicle of each multimarshalling MU train is selected as the research object. The results are shown in Figures 3-4 where abscissa shows the position of MU trains, that is, $0 \mathrm{~m}$ at the end of the bridge and $120 \mathrm{~m}$ of leaving the bridge, while the vertical coordinate is the standard deviation of the response of MU trains. From results, it can be seen that changes in the standard deviation of vehicle response are consistent and effects of marshalling types are mainly reflected in the magnitude of vehicle response. For the vehicles and bridge model applied into this paper, the total length of each vehicle model is $20 \mathrm{~m}$, the single is $40 \mathrm{~m}$, and the length of a single-span bridge can only 


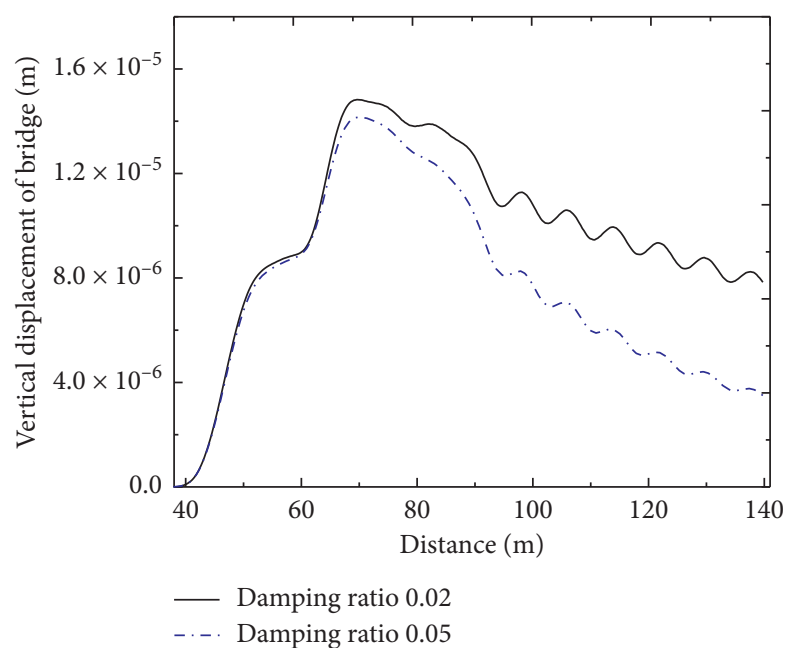

(a)

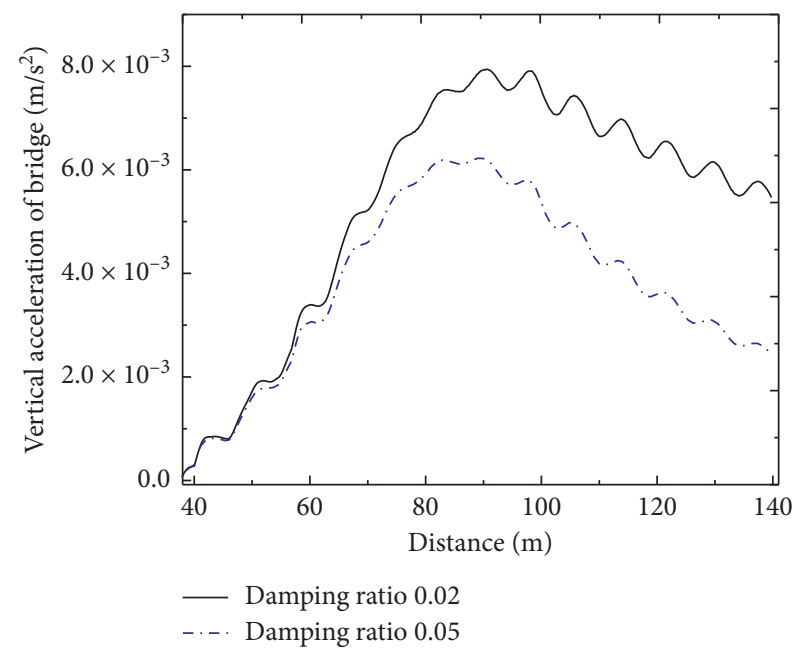

(c)

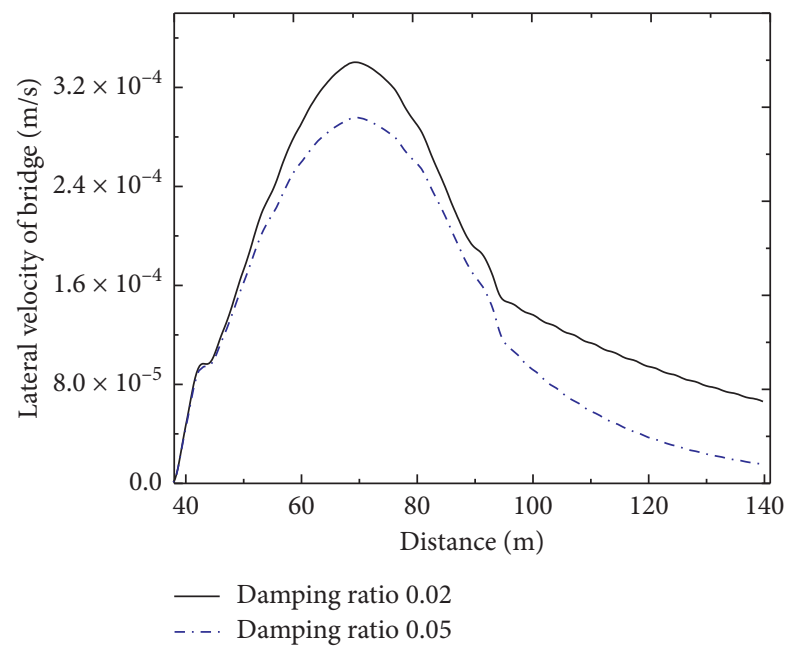

(e)

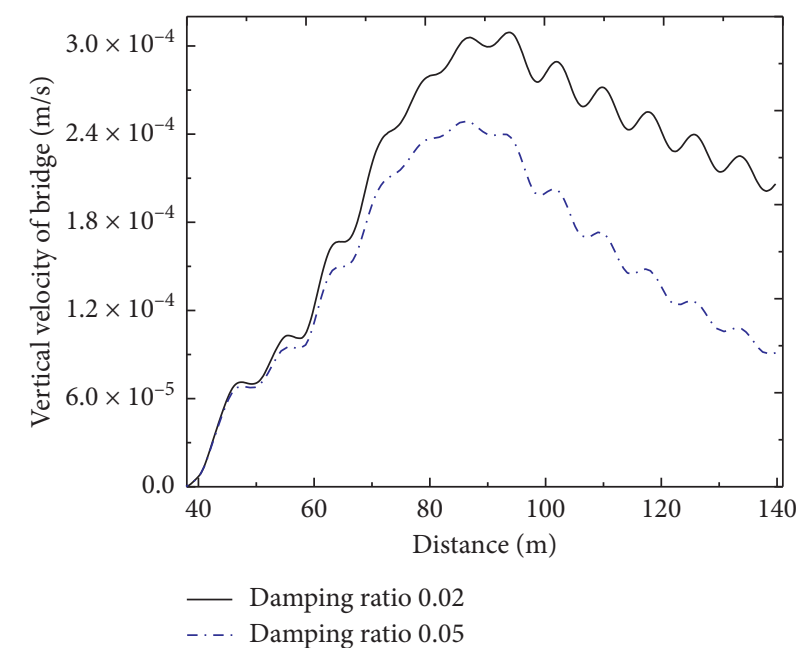

(b)

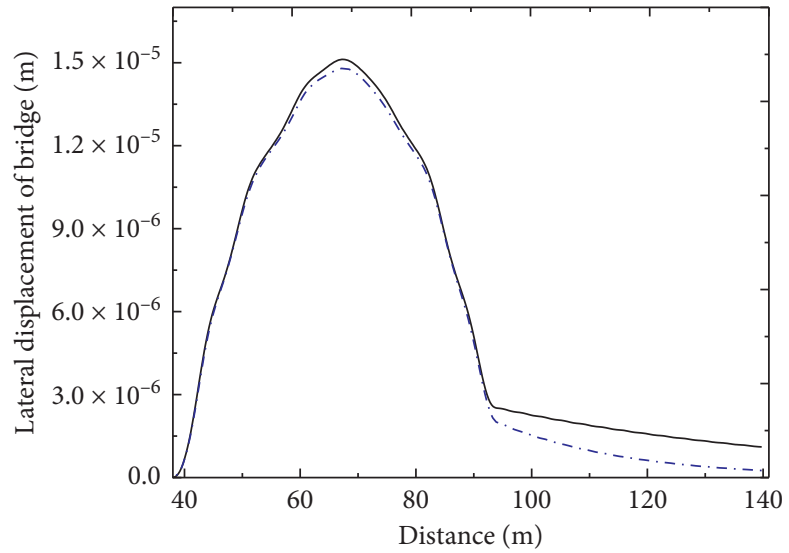

— Damping ratio 0.02

-... Damping ratio 0.05

(d)

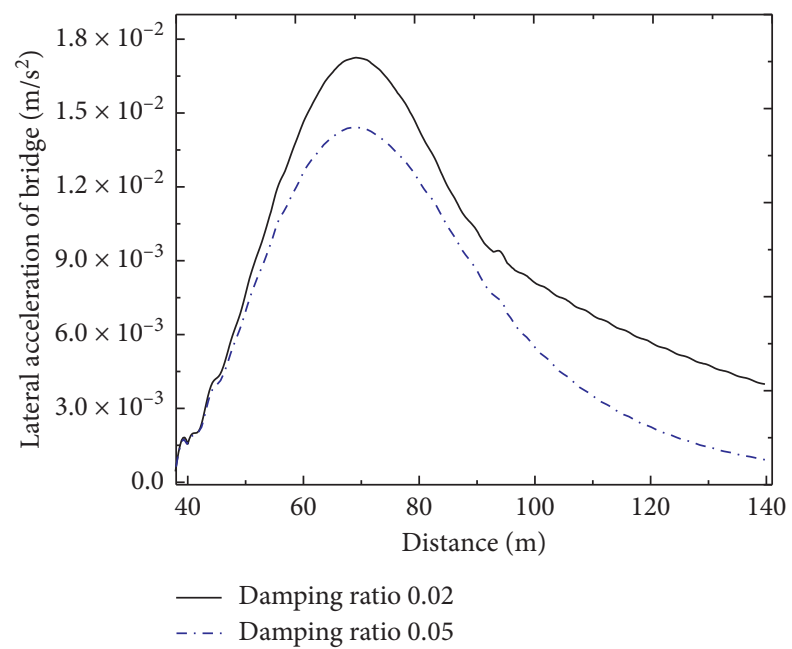

(f)

FIGURE 2: The mean square deviation of the bridge with different damping ratios.

contain two carriages at a time. Against the influence of track irregularity, the inertia force combination generated by vehicle vibration of each carriage forms continuous load with periodic variation that acts on the bridge structure, which makes the response of MU crossing the bridge distinctly different from that of a single-marshalling train 

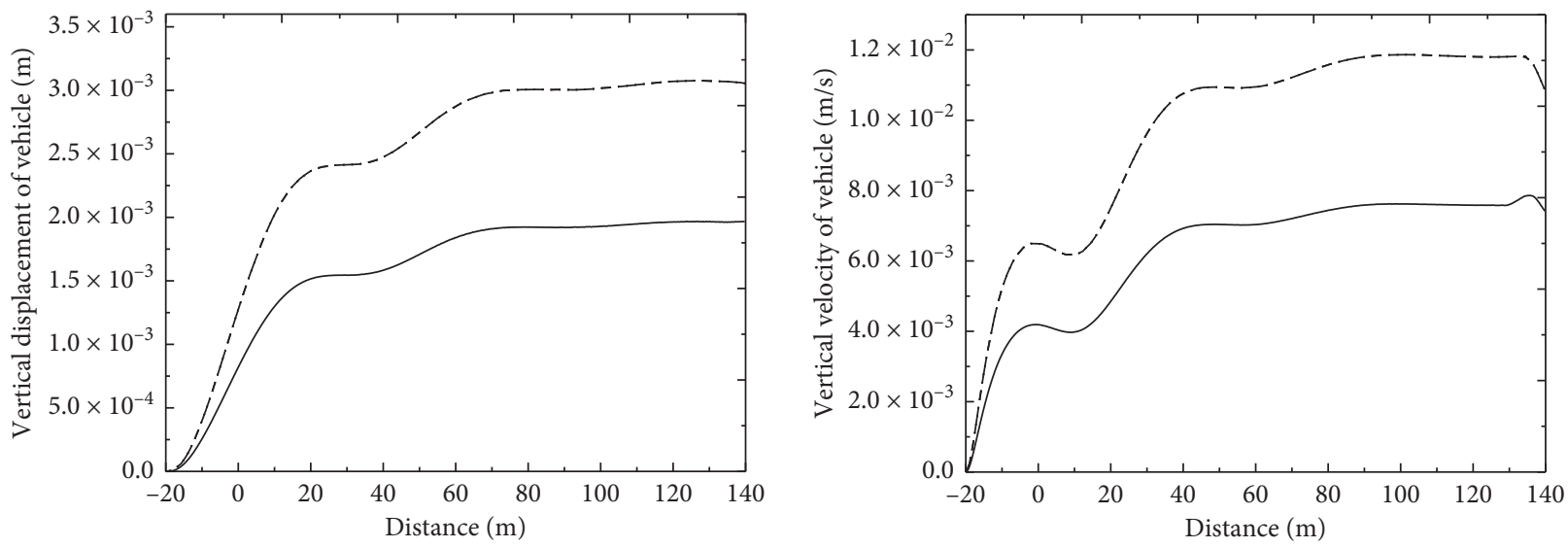

$\begin{array}{lll}\text { _ One vehicle } & \text {...... Four vehicles } \\ \text { - - Two vehicles } & \text {-... } & \text { Eight vehicles }\end{array}$

- One vehicle $\quad$...... Four vehicles

- - - Two vehicles

-.-. Eight vehicles

(a)

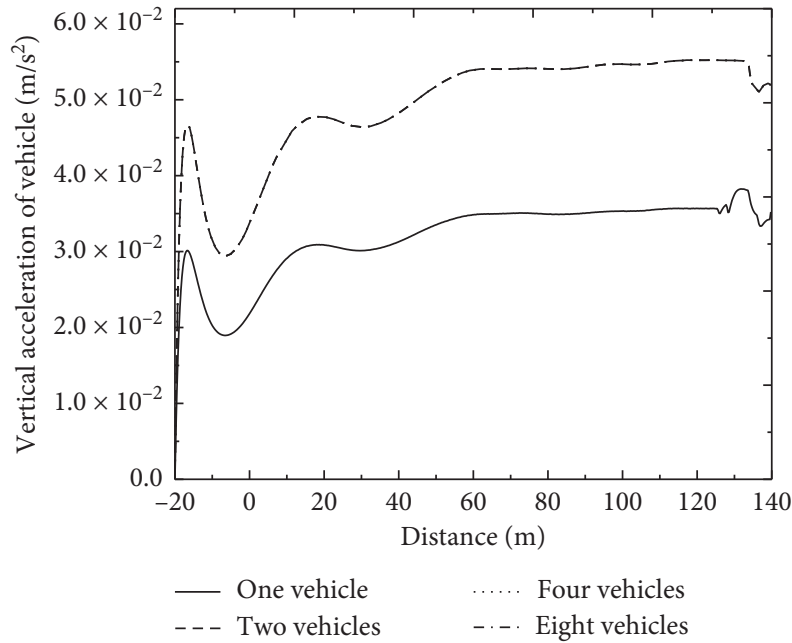

(c)

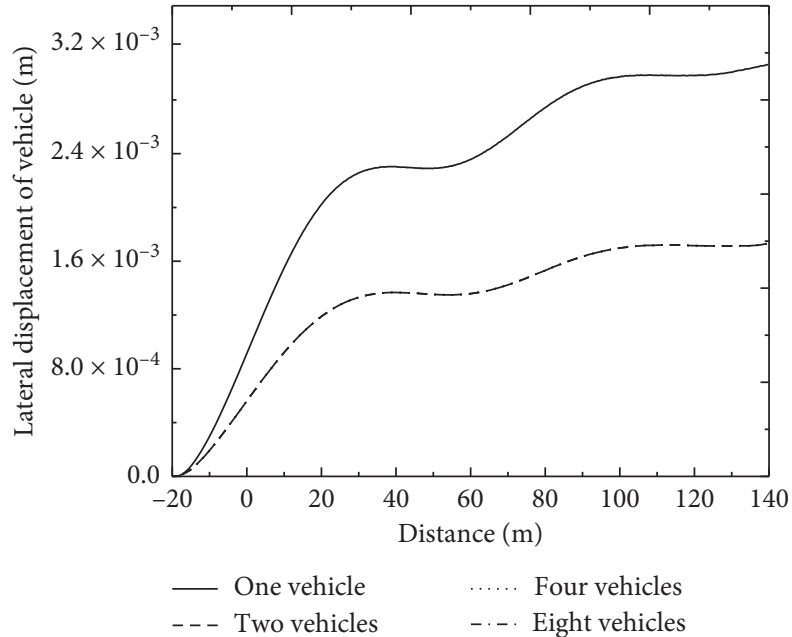

(d)

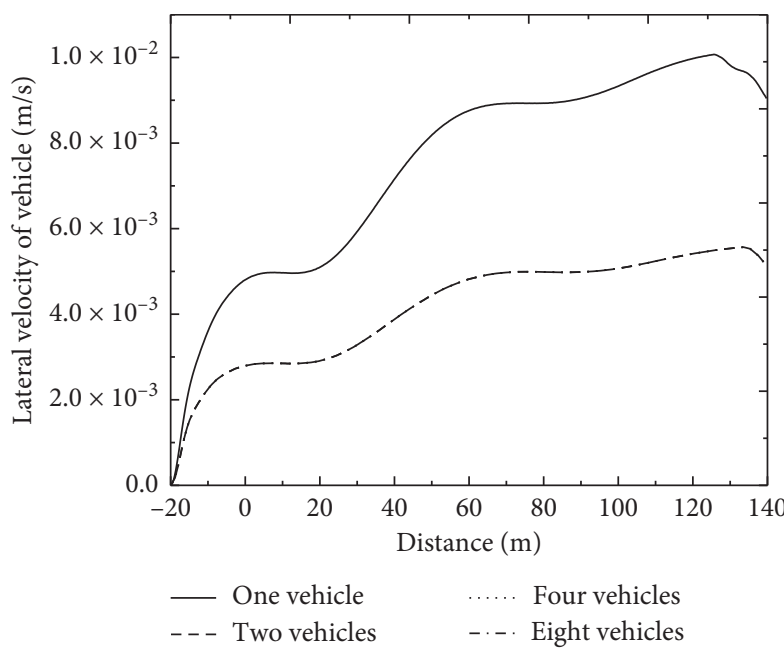

(e)

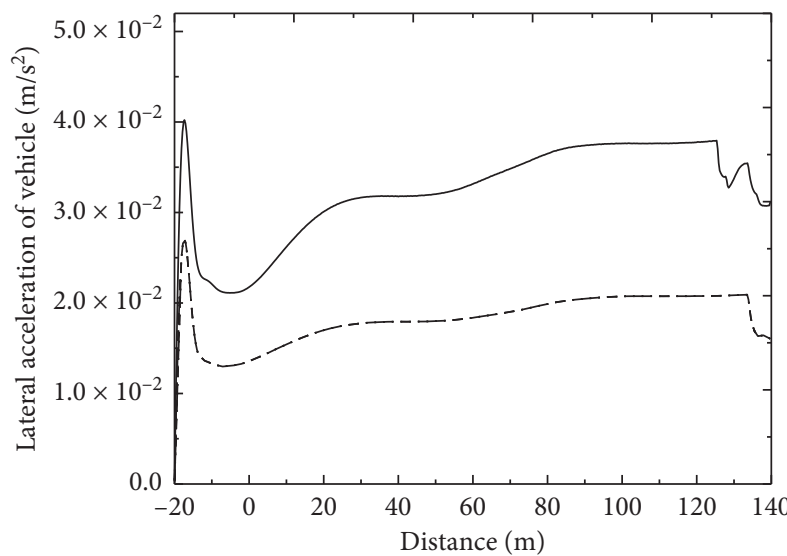

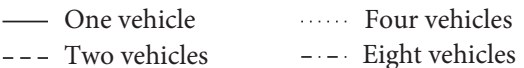

(f)

Figure 3: The mean square deviation of the vehicle with different train marshalling.

crossing the bridge. However, as shown in Figures 3-4, the standard deviation of response of the first train in doublemarshalling, four-marshalling, and eight-marshalling trains is basically coincident, which means that random characteristics of vehicle response do not change with the different marshalling account when the vehicle is on the bridge 


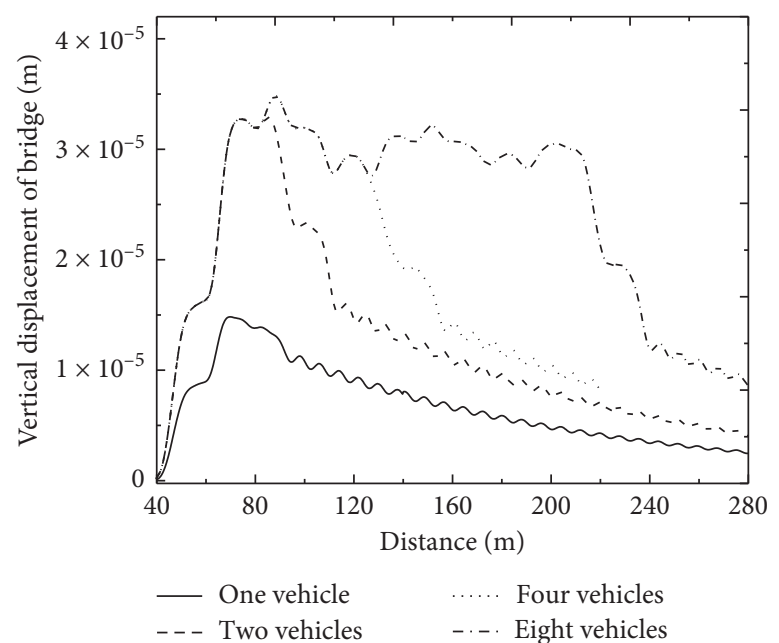

(a)

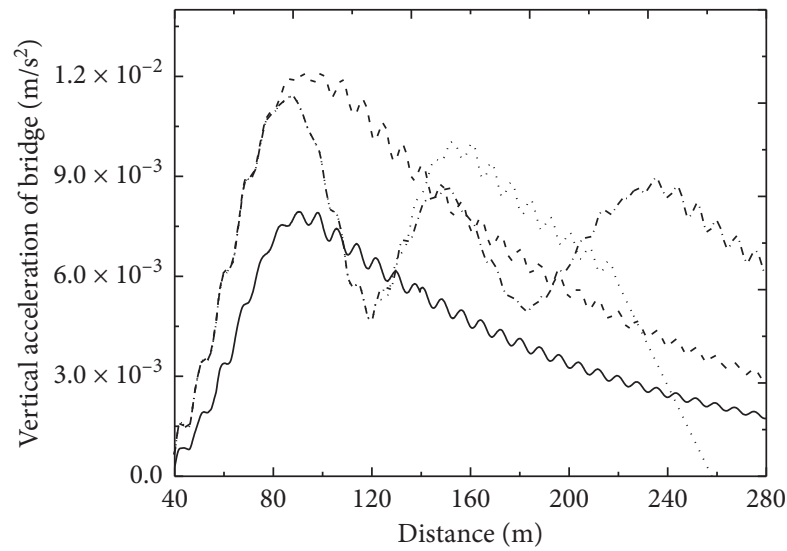

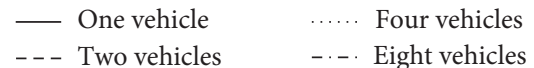

(c)

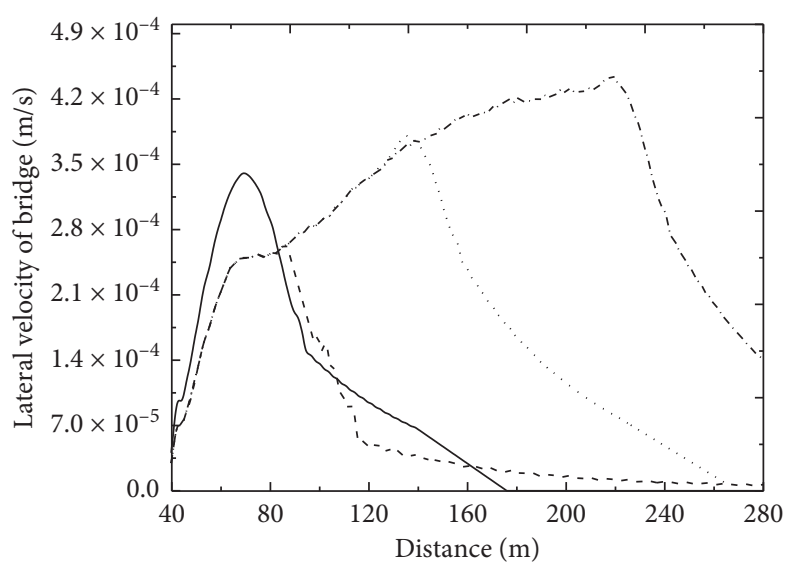

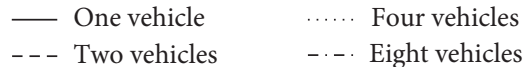

(e)

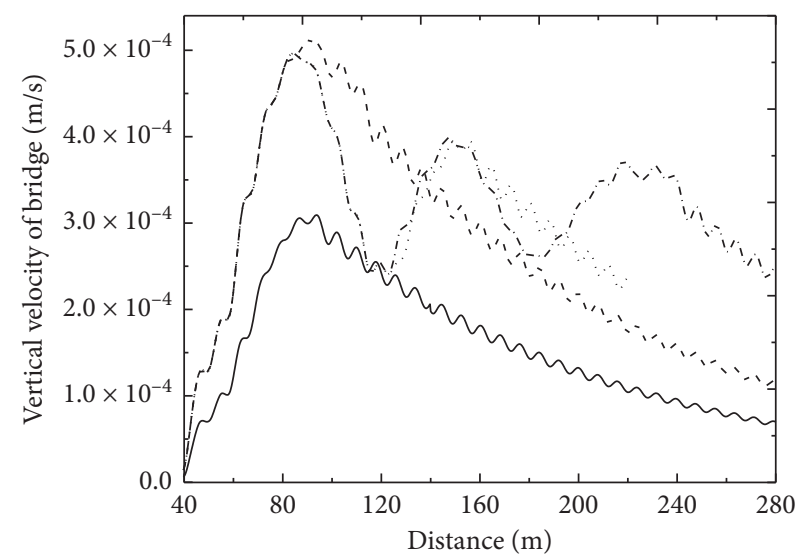

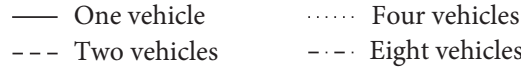

(b)

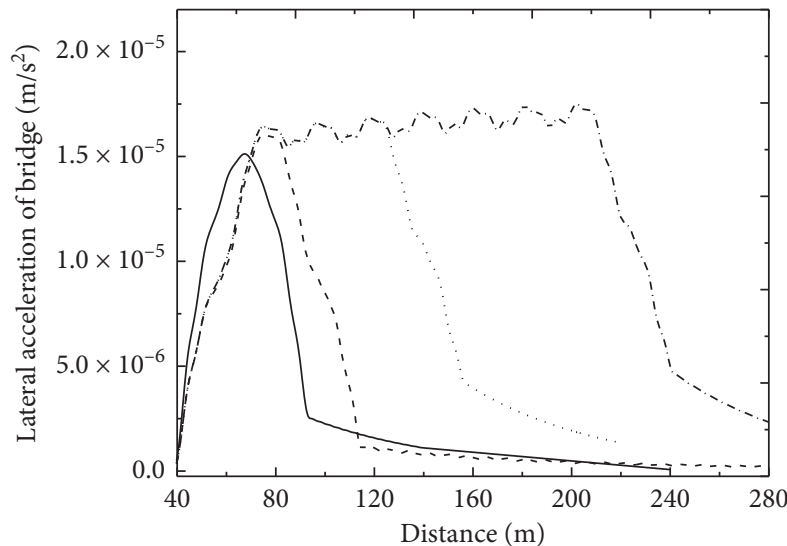

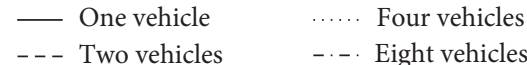

(d)

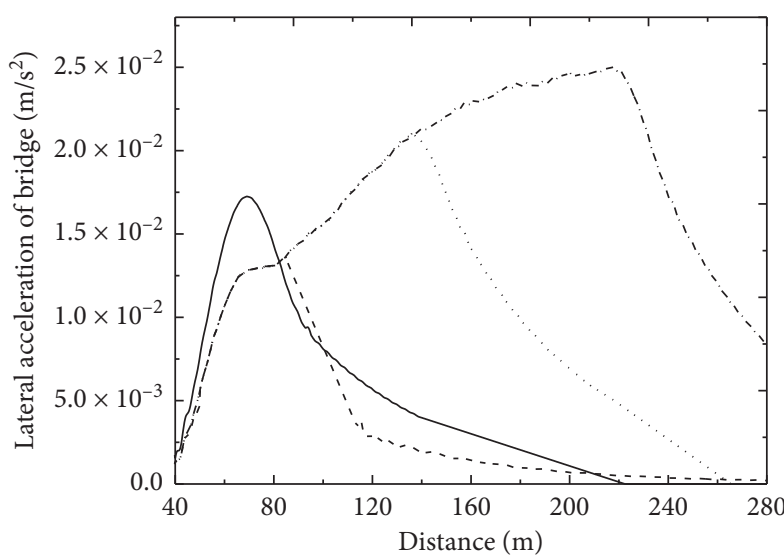

(f)

FIgURE 4: The mean square deviation of the bridge with different train marshalling.

completely. Taking the vertical acceleration of the vehicle body as an example, response of random vibration of MU trains is obviously larger than that of the single-marshalling train crossing the bridge. The standard deviation curves of these two models demonstrate a similar tendency that acceleration response becomes steady random process at the 
same position and the random vibration response of the vehicle will not be affected by marshalling. With combined effects of multimarshalling, the coupling force generated by vehicle vibration forms a periodic dynamic load acting on the bridge model, which increases the vertical vibration of the bridge and the random vibration response of the vehicle also increases sharply. Different from the vertical acceleration of the vehicle body, the periodic dynamic load formed by marshalling MU trains reduces the lateral random vibration response. During vehicle's moving, the lateral periodic load generated by the vehicle body can control the lateral dynamic vibration. The axle load of moving vehicles is the decisive factor leading to the dynamic vibration of the bridge, while the decisive factor of dynamic response of the vehicle is external excitation load. Therefore, random characteristics of vehicles are less affected by the dynamic vibration of the bridge. For the random vibration of vehicles, the random characteristics of response are less affected by marshalling types when the stiffness of the bridge structure is large.

The marshalling number of MU trains has a significant impact on the random characteristics of response of the bridge. Figure 4 shows that standard deviation of lateral displacement in the bridge span changes steadily when trains reach $74.74 \mathrm{~m}$, i.e., when MU trains are completely loaded on the simply supported girder bridge, demonstrating that the marshalling number does not affect the amplitude of the lateral displacement. Similarly, when MU trains are completely loaded on the simply supported girder bridge, standard deviation of the vertical displacement shows a quasisteady trend as standard deviation changes in a very small range. However, the vertical velocity and acceleration of bridge structure show a decreasing trend. It can be predicted that the vertical velocity and acceleration of bridge will gradually decrease and show a steady change in a certain range if the marshalling number increases. Additionally, as random characteristics of the lateral velocity and acceleration of the bridge show monotonic increase, the amplitude of standard deviation of response also increases. The standard deviation of the response of vertical displacement, vertical velocity, and vertical acceleration increase by nearly 1 time, 0.6 times, and 0.4 times, respectively. However, the amplitude of standard deviation of lateral response does not change significantly.

\section{Conclusions}

In this paper, a self-adaptive optimization algorithm, which combines self-adaptive Gauss integration with the pseudoexcitation method, is applied to investigate random vibration of the structure with different external loads. By selfadaptive iteration, the integral interval is subdivided into several reasonable integral subintervals and each integral subinterval is separately integrated by the Gauss numerical method, which greatly improves the efficiency of integration calculation. The proposed method can handle the random vibration of linear structure with random excitation, and the stationary excitation is calculated in this paper. The numerical examples show that self-adaptive Gauss integration technique can significantly reduce computational times and further improve computational efficiency of the pseudoexcitation method with the same requirements for accuracy. For the random vibration analysis of large-sized practical engineering structures, this method is of practical significance in engineering.

(1) Compared with the pseudoexcitation method, calculation times are only $10.6 \%$ of trapezoidal integration and the computational efficiency is increased by nearly 10 times in the application of the present method into the wind-induced random vibration analysis of structures

(2) The PEM-SGI is suitable to investigate the vibration of the vehicle-bridge coupling system. The structural damping ratio can effectively reduce the vibration of the bridge structure. Since the excitation frequency of the vehicle model is quite different from that of the bridge structure, the change of structural damping ratio cannot affect the random response of the vehicle model, but effectively control the dynamic response of the bridge structure.

(3) Due to the influence of MU trains' marshalling, inertial force generated by the vibration of the vehicle against the effect of track irregularity forms a periodic load acting on the bridge structure, which increases the standard deviation of the vertical response of vehicle models and bridge structure, but reduces the lateral standard deviation of vehicle models. With the influence of multimarshalling, the variance of displacement response of the bridge structure also shows some characteristics of regular random process.

\section{Data Availability}

The data used to support the findings of this study are included within the article.

\section{Conflicts of Interest}

The authors declare no potential conflicts of interest with respect to the research, authorship, and/or publication of this article.

\section{Acknowledgments}

The authors are grateful for the financial supports from the National Natural Science Foundation of China (under grant 51908076 and 51608193), the National Science Fund for Distinguished Young Scholars (51525804), and a project supported by the Scientific Research Fund of Hunan Provincial Education Department (16C0645).

\section{References}

[1] S. H. Cr, Random Vibration in Mechanical Systems, Academic Press, New York, NY, USA, 2nd edition, 1963.

[2] W. Q. Zhu, "Recent developments and applications of the stochastic averaging method in random vibration," Applied Mechanics Reviews, vol. 49, no. 10S, pp. S72-S80, 1996. 
[3] A. D. Kiureghian, "A response spectrum method for random vibration analysis of mdf systems," Earthquake Engineering \& Structural Dynamics, vol. 9, no. 5, pp. 419-435, 2010.

[4] S. Türkay and H. Akçay, "A study of random vibration characteristics of the quarter-car model," Journal of Sound and Vibration, vol. 282, no. 1-2, pp. 111-124, 2005.

[5] Y. H. Zhang, Q. S. Li, J. H. Lin, and F. W. Williams, "Random vibration analysis of long-span structures subjected to spatially varying ground motions," Soil Dynamics and Earthquake Engineering, vol. 29, no. 4, pp. 620-629, 2009.

[6] K. Soyluk, "Comparison of random vibration methods for multi-support seismic excitation analysis of long-span bridges," Engineering Structures, vol. 26, no. 11, pp. 15731583, 2004.

[7] A. Kareem, "Dynamic response of high-rise buildings to stochastic wind loads," Journal of Wind Engineering and Industrial Aerodynamics, vol. 42, no. 1-3, pp. 1101-1112, 1992.

[8] S. Benfratello, M. Di Paola, and P. D. Spanos, "Stochastic response of mdof wind-excited structures by means of volterra series approach," Journal of Wind Engineering and Industrial Aerodynamics, vol. 74-76, no. 98, pp. 1135-1145, 1998.

[9] Y. Li and A. Kareem, "Stochastic response of tension leg platforms to wind and wave fields," Journal of Wind Engineering and Industrial Aerodynamics, vol. 36, no. 1-3, pp. 915-926, 1990.

[10] T.-H. Le and L. Caracoglia, "Reduced-order wavelet-galerkin solution for the coupled, nonlinear stochastic response of slender buildings in transient winds," Journal of Sound and Vibration, vol. 344, pp. 179-208, 2015.

[11] J. Lin, W. Zhang, and F. W. Williams, "Pseudo-excitation algorithm for nonstationary random seismic responses," Engineering Structures, vol. 16, no. 4, pp. 270-276, 1994.

[12] J. H. Lin, Y. H. Zhang, and Y. Zhao, "Pseudo excitation method and some recent developments," Procedia Engineering, vol. 14, no. 2259, pp. 2453-2458, 2011.

[13] Y.-W. Zhang, Y. Zhao, Y.-H. Zhang, J.-H. Lin, and X.-W. He, "Riding comfort optimization of railway trains based on pseudo-excitation method and symplectic method," Journal of Sound and Vibration, vol. 332, no. 21, pp. 5255-5270, 2013.

[14] Y. L. Xu, W. S. Zhang, J. M. Ko, and J. H. Lin, "Pseudoexcitation method for vibration analysis of wind-excited structures," Journal of Wind Engineering and Industrial Aerodynamics, vol. 83, no. 1-3, pp. 443-454, 1999.

[15] J. H. Lin, Y. H. Zhang, Q. S. Li, and F. W. Williams, "Seismic spatial effects for long-span bridges, using the pseudo excitation method," Engineering Structures, vol. 26, no. 9, pp. 1207-1216, 2004.

[16] C. C. Caprani, "Application of the pseudo-excitation method to assessment of walking variability on footbridge vibration," Computers \& Structures, vol. 132, no. 1, pp. 43-54, 2014.

[17] D. M. Huang, L. D. Zhu, and W. Chen, "Covariance proper transformation-based pseudo excitation algorithm and simplified srss method for the response of high-rise building subject to wind-induced multi-excitation," Engineering Structures, vol. 100, pp. 425-441, 2015.

[18] O. Coussy, M. Said, and J.-P. Van Hoove, "The influence of random surface irregularities on the dynamic response of bridges under suspended moving loads," Journal of Sound and Vibration, vol. 130, no. 2, pp. 313-320, 1989.

[19] J. M. Rocha, A. A. Henriques, and R. Calçada, "Probabilistic safety assessment of a short span high-speed railway bridge," Engineering Structures, vol. 71, pp. 99-111, 2014.
[20] Z. P. Zeng, F. S. Liu, P. Lou, Y. G. Zhao, and L. M. Peng, "Formulation of three-dimensional equations of motion for train-slab track-bridge interaction system and its application to random vibration analysis," Applied Mathematical Modelling, vol. 40, no. 11-12, pp. 5891-5929, 2016.

[21] Z.-W. Yu, J.-F. Mao, F.-Q. Guo, and W. Guo, "Non-stationary random vibration analysis of a $3 \mathrm{D}$ train-bridge system using the probability density evolution method," Journal of Sound and Vibration, vol. 366, pp. 173-189, 2016.

[22] X. Yin, Z. Fang, C. S. Cai, and L. Deng, "Non-stationary random vibration of bridges under vehicles with variable speed," Engineering Structures, vol. 32, no. 8, pp. 2166-2174, 2010.

[23] J. Mao, Z. Yu, Y. Xiao, C. Jin, and Y. Bai, "Random dynamic analysis of a train-bridge coupled system involving random system parameters based on probability density evolution method," Probabilistic Engineering Mechanics, vol. 46, pp. 48-61, 2016.

[24] L. Wang, P. Jiang, Z. Hui, Y. Ma, K. Liu, and X. Kang, "Vehicle-bridge coupled vibrations in different types of cable stayed bridges," Frontiers of Structural and Civil Engineering, vol. 10, no. 1, pp. 81-92, 2016.

[25] L. Wang, X. Kang, and P. Jiang, "Vibration analysis of a multispan continuous bridge subject to complex traffic loading and vehicle dynamic interaction," KSCE Journal of Civil Engineering, vol. 20, no. 1, pp. 323-332, 2016.

[26] W. R. Fehlner, S. B. Nickerson, and S. H. Vosko, "Cubic harmonic expansions using gauss integration formulas," Solid State Communications, vol. 19, no. 1, pp. 83-86, 1976.

[27] B. Bojanov and G. Petrova, "Numerical integration over a disc. A new Gaussian quadrature formula," Numerische Mathematik, vol. 80, no. 1, pp. 39-59, 1998.

[28] F. Curbera, "Delayed curse of dimension for Gaussian integration," Journal of Complexity, vol. 16, no. 2, pp. 474-506, 2000.

[29] S. Zhu, Y. Li, K. Togbenou, and T. Xiang, "An efficient optimization algorithm to study the stochastic responses of vehicle-bridge coupling system," Computing in Science \& Engineering, vol. 21, no. 3, pp. 6-17, 2018.

[30] J. H. White, "Self-linking and the gauss integral in higher dimensions," American Journal of Mathematics, vol. 91, no. 3, pp. 693-728, 1969.

[31] M. Head-Gordon and J. A. Pople, "A method for two-electron Gaussian integral and integral derivative evaluation using recurrence relations," The Journal of Chemical Physics, vol. 89, no. 9, pp. 5777-5786, 1988.

[32] A. G. Davenport, "The application of statistical concepts to the wind loading of structures," Proceedings of the Institution of Civil Engineers, vol. 19, no. 4, pp. 449-472, 1961. 


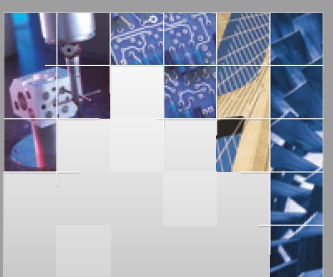

\section{Enfincering}
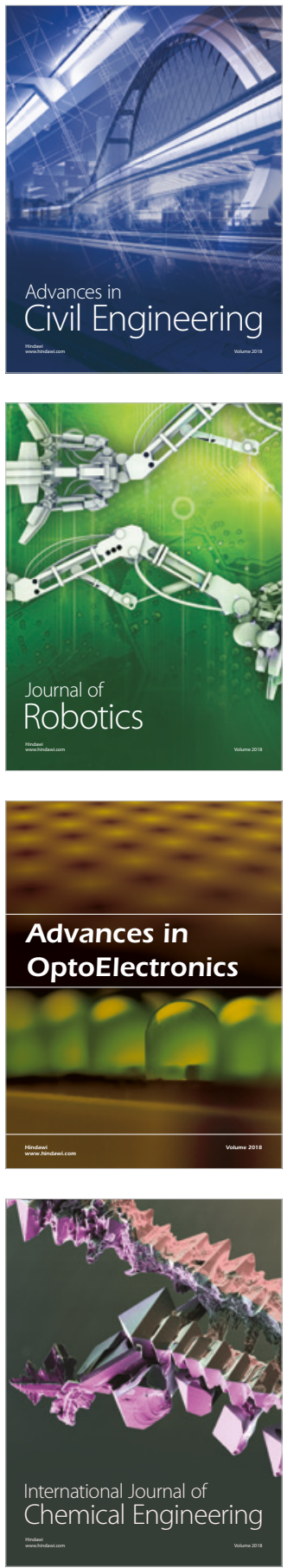

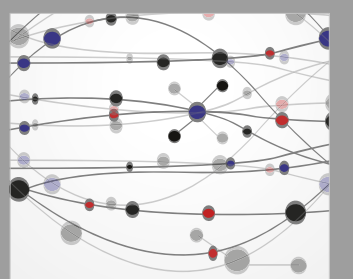

\section{Rotating \\ Machinery}

The Scientific World Journal

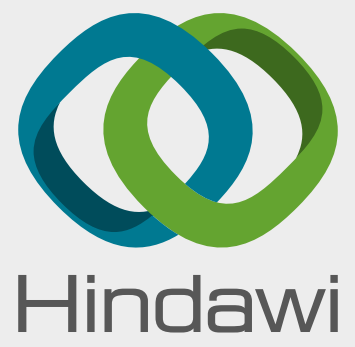

Submit your manuscripts at

www.hindawi.com
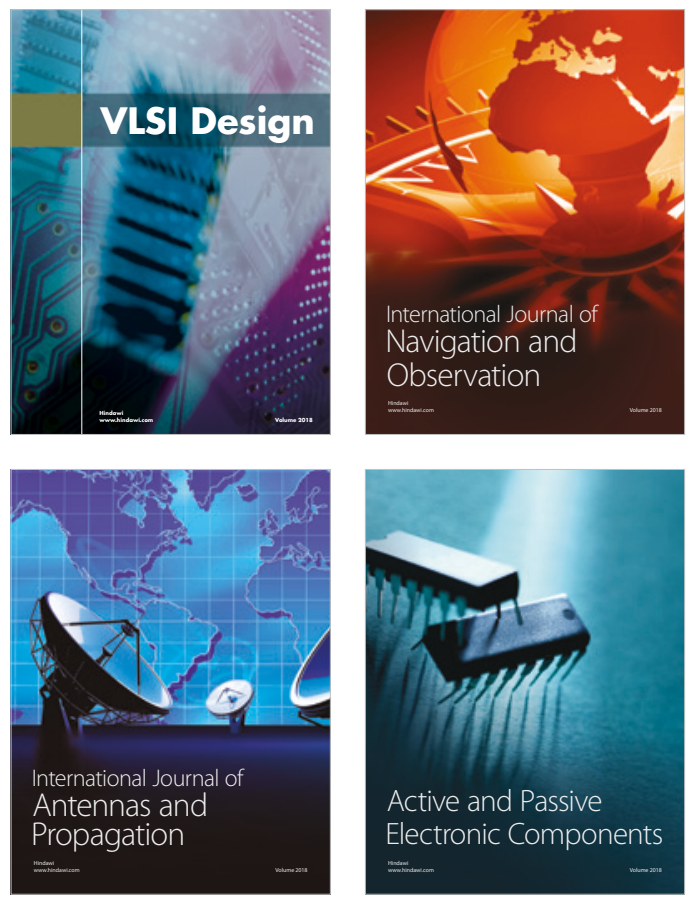
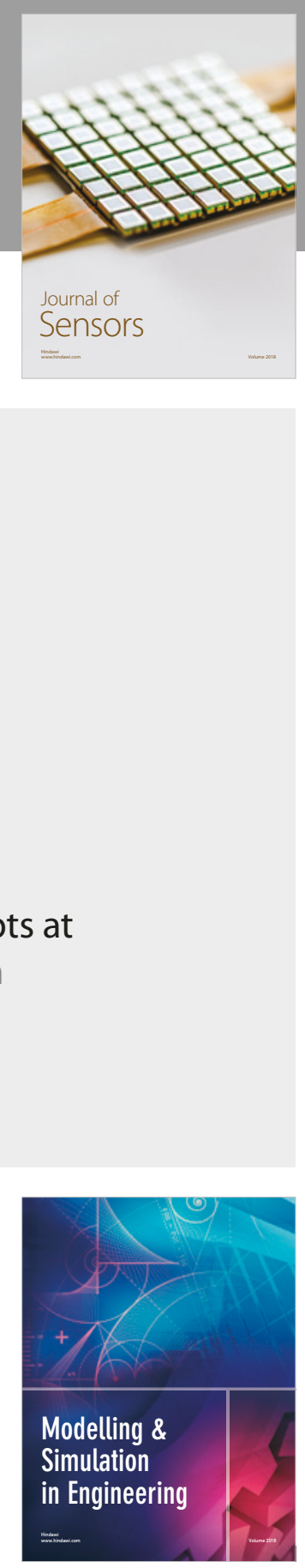

\section{Advances \\ Multimedia}
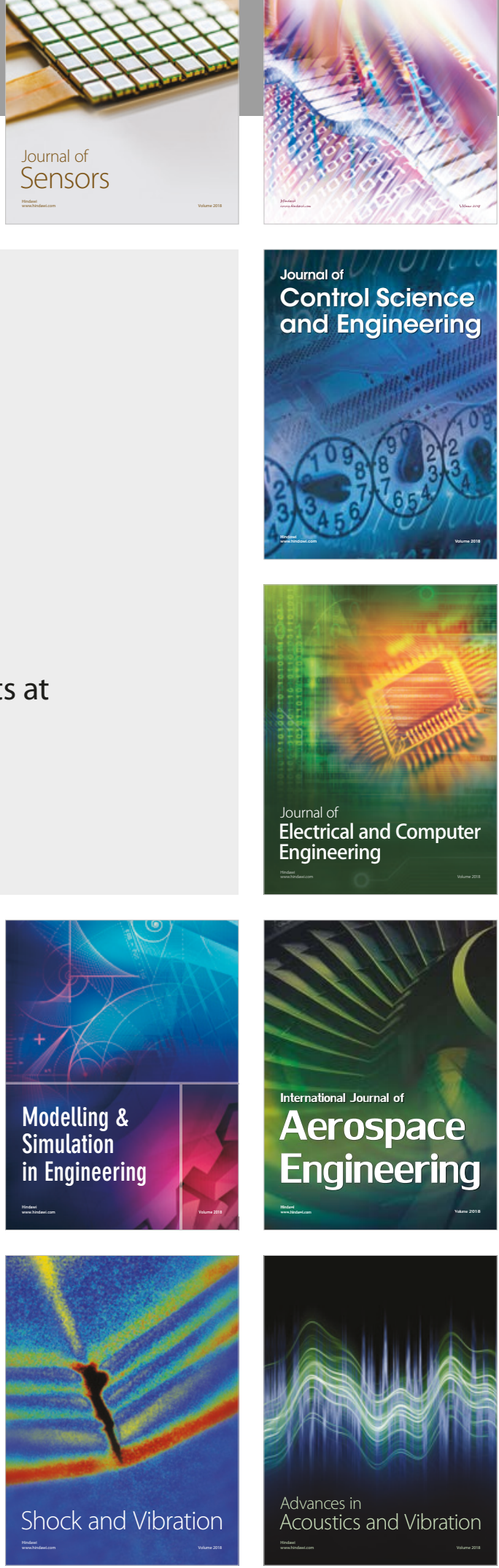